\title{
Central venous catheter-related blood stream infections in a neonatal care unit
}

\author{
Rawia Ibrahim Badr', Enas Hammad', Mona Foda Salama', \\ Basma Shouman', Hesham Abdel-Hady', Nehad Nasef ${ }^{2}$
}

1. Microbiology and medical Immunology Department Mansoura University, Mansoura, Egypt 2. Neonatal Care Unit, Faculty of Medicine, Mansoura University, Mansoura, Egypt

\begin{abstract}
The aim of this study was to detect the rate of catheter-related bloodstream infection (CRBSI) in NICU of Mansoura University Children's Hospital and to focus on possible predictors of infection. A cross sectional comparative study was performed to detect the rate of CRBSI among neonates with central vascular catheters during the period between January 2009 and April 2012. Then, a nested case control study done among CRBSI cases and central vascular catheters none infected as a control group to detect predictors associated with their occurrence. Blood samples were collected from 111 neonates clinically suspected of septicemia and had inserted venous line. Central, peripheral blood and catheter tip cultures were done for each case.

CRBSI was confirmed in 69 cases as central blood culture count was more than 3 folds of peripheral blood culture count. Catheter tip culture showed more than $103 \mathrm{CFU} / \mathrm{ml}$ per catheter of the same organisms. Low birth weight, age below 7 days, prematurity, mechanical ventilation, TPN administration and prolonged catheter stay were predictors of CRBSI. Logistic regression of the studied predictors showed that birth weight, TPN administration and length of central venous catheter stay were significant predictors of CRBSI. CRBSI is a common problem in NICU. Predictors of CRBSI included low birth weight, prematurity and mechanical ventilation. Potential use of TPN, prolonged catheter stay and low birth weight are independent predictors for CRBSI.
\end{abstract}

Keywords: Catheterization, central venous; Intensive care units, neonatal; Cross infection; Catheter-related infections

\section{Corresponding Author}

Rawia Ibrahim Badr

Assistant Professor of medical Microbiology and Immunology

Faculty of medicine, Mansoura University, Mansoura, Egypt

Email: rawbadr@gmail.com 


\section{Introduction}

Health care-associated blood stream infections (HABSI) among infants hospitalized in the neonatal intensive care unit (NICU) are a leading cause of morbidity and mortality. Neonatal health care associated infections are often related to the use of central venous catheters (CVC) which are necessary to administer intravenous fluids and medications. ${ }^{1}$

Catheter-related blood stream infection (CRBSI) is categorized into septic, exit site, and tunnel infections. Infection is diagnosed on the basis of local inflammation, purulence, fever, and leukocytosis. Lack of localizing signs and debilitation among patients with central lines attribute to difficulty to diagnose septic infections. ${ }^{2}$

Although the risk of death from CRBSI is low, infection of intravascular devices is today's leading cause of nosocomial bacteremia. ${ }^{3,4,5}$ Fever is the most common clinical sign. Others include chills associated with catheter flushing. ${ }^{6}$ CRBSI can be diagnosed when colony counts are at least 3-times higher in cultures of blood obtained via the CVC than in cultures of blood taken from a peripheral vein. ${ }^{7}$

If the CVC is removed for suspected CRBSI, the catheter tip should be submitted for culture. The port reservoir contents from removed subcutaneous port should be cultured. If the organisms present in either culture have also been isolated from a percutaneously obtained blood culture, CRBSI is confirmed. ${ }^{7}$ This study aimed to detect the rate of CRBSI and its possible predictors in the NICU.

\section{Subjects \& Methods}

\section{Study design}

This is a cross sectional comparative study carried out to detect rate of CRBSI among neonates with CVC. Then, a nested case control study was done among CRBSI cases and CVC non infected as a control group to detect the predictors associated with their occurrence including sex, weight at birth, total parenteral nutrition (TPN), neonatal age at catheter insertion, weeks of gestation, length of catheter stay, number of previously inserted CVC and mechanical ventilation. Stepwise logistic regression model were then fit using predictors significant in the bivariate analyses. The National Health Safety Network (NHSN) definition and reporting of CRBSI was applied. ${ }^{1}$

\section{Study population and Inclusion criteria}

This study was conducted in the NICU (22 incubators), of Mansoura University Children's Hospital (MUCH) in conjunction with Microbiology Diagnostics Infection Control Unit (MDICU) in Medical Microbiology and Immunology Department in Mansoura Faculty of Medicine starting from January 2009 until April 2012. The study enrolled neonates with CVC. Blood samples from clinically suspected septicemic patients were submitted for diagnosis of CRBSI.

History and physical examination were recorded using a standardized form with special concern for evidence of risk factors. Written consent from the parents precede enrollment in the study. The study was approved by the institutional review board of $\mathrm{MUCH}$.

\section{Methodology}

\section{Catheters insertion technique}

Percutaneous central venous catheterization including peripherally inserted central catheters (PICC line) or umbilical venous catheters were placed in neonates for long-term venous access for intravenous fluids, particularly parenteral nutrition. PICC lines were preferentially used in low birth weight infants. The operator and staff assisting with catheter insertion wore a surgical mask, perform a surgical hand wash with povidone-iodine containing soap (Isobetadine, Asta Medica, Brussels, Belgium) wore a sterile gown and sterile gloves. Percutaneous placement of a CVC was performed at the bedside by the neonatologist under strict aseptic conditions. After local skin disinfection with a solution of povidone-iodine solution and $0.5 \%$ chlorhexidine in $70 \%$ isopropanol (Hibitane solution, Zeneca), the introducer needle is inserted into a vein e.g. basilic, greater saphenous, external jugular or subclavian vein then the catheter is threaded and advanced the predetermined distance for central venous positioning. The catheter tip is positioned at the junction of the vena cava and right atrium, as confirmed by radiography. ${ }^{8}$ 
In neonates who had umbilical venous catheters, a 3.5 or 5 French catheter was inserted in the umbilical vein, advanced through the ductus venosus into the inferior vena cava and its position verified by $\mathrm{x}$-ray. ${ }^{8}$ The catheters were left in place until no longer needed or until a complication such as bacteremia, venous thrombosis or catheter malfunction occurred.

\section{Sample collection and processing}

When CRBSI was suspected, the catheter hub was cleaned with alcohol and allowed to dry to minimize blood culture contamination; 1-2 $\mathrm{ml}$ of blood was drawn from lumen of CVC for quantitative blood culture by the pour plate method on nutrient agar plates after serial dilution. Colonies were formed on the top of the agar as well as in the agar, those on top, were larger but all colonies on and in the agar were counted. Plates that had between 30 - 300 colonies in and on the agar were selected as those give the best statistical representation of the number of bacteria in the undiluted sample. Quantitative culture was reported according to the following equation $\mathrm{CFU} / \mathrm{ml}$ $=\mathrm{CFU} /$ plate $\mathrm{x}$ dilution factor.

Simultaneous quantitative peripheral blood culture from a fresh venipuncture was done taking all aseptic precautions. Media used for subculture included chocolate agar, $5 \%$ sheep blood agar and MacConkey's agar (Oxoid, UK).

After removal, the tip of the CVC was sent for culture. and inoculated agar plates were incubated at $37^{\circ} \mathrm{C}$ for up to 48 hours Results were reported as positive if $\geq 10^{3} \mathrm{CFU} / \mathrm{ml}$ of broth cultures per catheter were counted on the agar plate.

\section{Microbial identification}

Full identification up to species level was done by Gram stained film, colony characters, and biochemical reactions. API 20E and API 20 non E (bioMerieux, Marcy l'Etoile, France) was used to confirm the identity of Gram negative isolates. While culture characters, catalase, coagulase and API 20 Staph were used to identify Gram positive isolates.

\section{Antimicrobial Susceptibility Tests}

Susceptibility tests were performed using the KirbyBauer disc diffusion method on the surface of Muller-
Hinton agar plates (Oxoid, UK) following National Committee for Clinical Laboratory Standards (NCCLS) guidelines. ${ }^{9}$

\section{Plasmid extraction}

Cases proven to be CRBSI, Plasmid extraction was carried out to ensure that they have the same identity of those isolated from peripheral blood culture. Briefly one and half $\mathrm{ml}$ of broth culture of the organism was centrifuged at 12,000 rpm for 30 seconds at $4^{\circ} \mathrm{C}$. Then, the bacterial pellet was resuspended in $100 \mu$ l of ice cold solution I [50 mM glucose, $25 \mathrm{mM}$ TrisCL $(\mathrm{pH}$ 8.0), $10 \mathrm{mM}$ EDTA ( $\mathrm{pH} 8.0$ )] and vigorously vortexed. Two hundred $\mu \mathrm{l}$ of freshly prepared solution II $[0.2$ $\mathrm{NaOH}$ in $1 \%$ SDS] were added and inverted rapidly five times and stored on ice for 5 minutes. Then, one hundred and fifty $\mu \mathrm{l}$ of ice cold solution III [3M K+ acetate in $11.5 \%$ glacial acetic acid] were added and vortexed for 10 seconds then stored on ice for 3-5 minutes. Next, supernatant was collected after centrifugation at $12,000 \mathrm{rpm}$ for 5 minutes at $4^{\circ} \mathrm{C}$. Two volumes of ethanol were added, vortexed and left at room temperature for 2 minutes. The tubes were centrifuged at $12,000 \mathrm{rpm}$ for 5 minutes at $4^{\circ} \mathrm{C}$. After removal of the supernatant, pellets were rinsed with $1 \mathrm{ml}$ of $70 \%$ ethanol then the products were visualized under ultraviolet light after electrophoresis on $2 \%$ agarose gel stained with ethidium bromide. The plasmid pattern and size con were compared with standard molecular weight marker. ${ }^{10}$

\section{Statistical analysis}

Analysis of data was undertaken using SPSS (statistical package for social science) version 16 . Variables were presented as number and percentage. Chi-square test was used for comparison between groups. Significant predictors on bivariate analysis were entered into a logistic regression model using forward Wald method to predict the independent predictors of infection. Odds ratio $(\mathrm{OR})$ and confidence interval $(95 \% \mathrm{Cl})$ were calculated. The significance were adopted when $\mathrm{p}<0.05$.

\section{Results}

Over the period of this study, 1314 neonates admitted to NICU unit of whom, 293 (22.3\%) had one CVC line inserted, including 192 (65.5\%) umbilical venous catheters, 77 (26.7\%) CVC and 24 (8.2\%) 
PICC lines. Blood samples were collected from 111 neonates suspected to have septicemia, CRBSI was detected in $69(23.5 \%)$ patients guided by culture and identification. The overall infection rate for CRBSI was 21.4 per 1,000 CVC days commonly caused by CoNs $(n=41), K$. pneumoniea $(n=25)$, S.aureus $(n=3), E$. cloacae $(\mathrm{n}=2)$ and $P$. aeruginosa $(\mathrm{n}=1)$. Mixed CoNs, S. aureus, and Enterococcus were detected in 2 cases.

CoNS and S. aureus that were isolated from blood samples through the CVC shared $100 \%$ sensitivity to vancomycin while Klebsiella isolates were only sensitive to imipenem (93.4\%). Enterobacter isolates showed best sensitivity to meropenem (100\%) followed by ciprofloxacin (88.9\%). Pseudomonas were most susceptible to imipenem (98.2\%) followed by ceftazidime ( $88 \%$ ) and gentamycin $(64.7 \%)$.

CRBSI was confirmed in 69 cases. Catheter tip culture showed more than $10^{3} \mathrm{CFU} / \mathrm{ml}$ per catheter of the same organisms. Further confirmation by biotyping, resistogram and plasmid typing were done. Three clinically suspected septicaemic cases with positive culture results were excluded from the study, two cases did not match diagnostic criteria for CRBSI. The remaining case was positive only by catheter tip culture. All cultures from the remaining 39 clinically suspected cases were negative.

Table I. Predictors of catheter-related versus non catheter-related BSI

\begin{tabular}{|c|c|c|c|}
\hline Risk factors & $\begin{array}{r}\text { CRBSI } \\
(n=69) \\
\end{array}$ & $\begin{array}{r}\text { Non-CRBSI (control) } \\
(\mathrm{n}=224)\end{array}$ & $P$ \\
\hline \multicolumn{4}{|l|}{ Sex: } \\
\hline Male & $41(59.4 \%)$ & $118(48.8 \%)$ & \\
\hline Female & $28(40.6 \%)$ & $124(51.2 \%)$ & 0.077 \\
\hline \multicolumn{4}{|l|}{ Birth weight: } \\
\hline$\geq 1500 \mathrm{~g}$ & $13 \quad(18.8 \%)$ & $153(63.2 \%)$ & \\
\hline$<1500 \mathrm{~g}$ & $56 \quad(81.2 \%)$ & $89(36.8 \%)$ & 0.0001 \\
\hline \multicolumn{4}{|l|}{ TPN: } \\
\hline YES & $59 \quad(85.5 \%)$ & $108(55.4 \%)$ & \\
\hline $\mathrm{NO}$ & $10(14.5 \%)$ & $134(44.6 \%)$ & $<0.001$ \\
\hline \multicolumn{4}{|l|}{ Neonatal age: } \\
\hline$<7$ days & $38(55.1 \%)$ & $101(41.7 \%)$ & \\
\hline$\geq 7$ days & $31(44.9 \%)$ & $141(58.3 \%)$ & 0.034 \\
\hline \multicolumn{4}{|c|}{ Weeks of gestation: } \\
\hline$<34$ weeks & $66(95.7 \%)$ & $107(44.2 \%)$ & \\
\hline$\geq 34$ weeks & $3(4.3 \%)$ & $135(55.8 \%)$ & $<0.001$ \\
\hline \multicolumn{4}{|c|}{ Length of catheter stay: } \\
\hline$\geq 3$ days & $54(78.3 \%)$ & $69(28.5 \%)$ & \\
\hline$<3$ days & $15(21.7 \%)$ & $173(71.5 \%)$ & $<0.001$ \\
\hline \multicolumn{4}{|c|}{ Previously inserted CVC: } \\
\hline Yes & $20(29 \%)$ & $56(23.1 \%)$ & \\
\hline No & $49(71 \%)$ & $186(76.9 \%)$ & 0.20 \\
\hline \multicolumn{4}{|c|}{ Mechanical ventilation: } \\
\hline Yes & $41(59.4 \%)$ & $108(44.6 \%)$ & \\
\hline No & $28(40.6 \%)$ & $134(55.4 \%)$ & 0.04 \\
\hline
\end{tabular}

CRBSI: Catheter-related blood stream infection

CVC: Central venous catheter

TPN: Total parenteral nutrition 
Table II. Logistic regression model for risk factors

\begin{tabular}{lrrrrr} 
& B & Significance & OR & \multicolumn{2}{c}{$\mathbf{9 5 . 0 \%}$ C.I.for EXP(B) } \\
\cline { 4 - 6 } & & & & Lower & Upper \\
\hline Weight & -3.631 & $<0.001$ & 0.026 & 0.009 & 0.081 \\
\hline TPN & -1.326 & 0.012 & 0.265 & 0.095 & 0.744 \\
\hline Length of CVC insertion & -3.480 & $<0.001$ & 0.031 & 0.011 & 0.090 \\
\hline Constant & 3.274 & $<0.001$ & 6.409 & & \\
\hline
\end{tabular}

Model $\mathrm{x}^{2}=22.17, \mathrm{P}<0.01$

This model predicts $91 \%$

Neonates with CRBSI were more likely to be VLBW, aged below 7 days, premature, mechanically ventilated, on TPN, and had a longer catheter stay when compared with those without BSI. The risk of BSI did not differ by gender or previous catheterization (table I).

Logistic regression of the studied predictors showed that birth weight, TPN infusion and length of CVC stay were still significant predictors of CRBSI (table II).

\section{Discussion}

CVC is mandatory in the critically ill child including VLBW (VLBW), infants with short-gut syndrome, children with cancer, and those with chronic diseases such as cystic fibrosis and hemophilia. ${ }^{11}$ Complications with CVC are relatively common and result in increased morbidity, hospitalization, and costs. ${ }^{12}$

Throughout the period of the 40 months study, a total of 69 positive blood cultures were proven to be related to the inserted CVC at a percentage of $23.5 \%$ and 21.4 per 1,000 CVC days. These results were concordant with the data of other publications. ${ }^{13-17}$ This was in contrast, to the data reported in the NHSN report in which the CRBSI ranged from 3.1-6.4 per 1,000 CVC days. ${ }^{18}$

This high rate could be related to increased number of VLBW of our studied group who needed frequent handling and mechanical ventilation. Moreover, underlying patient condition, frequent changes in nurses which lead to lack of regularity of following the written guidelines for access and care of central venous catheters, or the physical environment have shared to this high rate. ${ }^{17,19}$

There is a wide range of disparity in the infection rates in different studies which may depend on unitassociated factors such as size and settings, patientassociated factors such as type of illness and its severity and catheter-associated factors such as type of catheter, site of insertion and conditions under which the catheter was located. ${ }^{20}$ Stover et al. reported great inconsistency in the blood stream infections rates from 23 US hospitals ranging from 0.0 to $18.5 / 1000$ catheter-days. ${ }^{15}$ Richard et al. examined BSI in the USA and found that blood stream infection in 61 PICUs to be the most common nosocomial infection with a rate ranging from 0.0 to $20 / 1000$ catheter-days. ${ }^{21}$

In one study, CRBSI; only central venous catheters type was detected at a rate of 9.3 infections per 1,000 catheter days which is lower than our reported rate. ${ }^{22}$ Another prospective study of 226 neonates who had PICCs in NICU between January 2006 and June 2009, the PICC infection rate was 17 per 1000 catheterdays. ${ }^{23}$ This could be explained by that, the CRBSI were not categorized according to type of catheters in this study.

In another report, the incidence of CRBSI was classified according to the neonatal birth weight to be 11 per 1000 catheter-days and 4 per 1000 catheter-days in babies weighing less than $1 \mathrm{k}$ and in those more than $2.5 \mathrm{~kg}$, respectively. ${ }^{24}$ 
CoNs (59.4\%) were the most common pathogen isolated from positive cultures as has been reported by other authors. ${ }^{19,25}$ The high frequency of this organism in CRBSI is due to its ability to adhere to the exterior surface of the catheter and formation of biofilm and slime layer which protects them from phagocytosis and antibiotics. ${ }^{14}$ Furthermore, the presence of lipid emulsion in the infusate has been linked to CoNS bacteremia. ${ }^{26}$

An alarming point in this study is the emergence of higher incidence of Klebsiella (36.2\%) among the organisms causing CRBSI than other previously mentioned data. ${ }^{17,23,27}$ The most common pathogens of bacterial sepsis differ in various parts of the world and health settings. This high incidence could be due to the fact that Klebsiella is a major pathogen in our NICU.

In the study carried in NICU in MUCH, Klebsiella was the commonest organism (46.2\%) isolated from blood stream infected cases. ${ }^{28}$ Also Rizk et al. found that Klebsiella was the most frequent pathogen isolated in MUCH accounting for $17.0 \%$ of the total nosocomial isolates. ${ }^{29}$ Another study was done in $\mathrm{MUCH}$ pediatric ICU revealed that Klebsiella was the commonest isolated nosocomial organism. ${ }^{30}$ In addition, Badr and Shouman found that Klebsiella was responsible for nearly $50 \%$ of neonatal sepsis in MUCH NICU. ${ }^{31}$ Moreover, Klebsiella was found to be the commonest isolate among admitted neonates in the same $\mathrm{NICU}$ representing $39.2 \%{ }^{32}$

Hospitalized neonates are often colonized with Klebsiella, Enterobacter, and Citrobacter species at high rates when compared with babies at home in whom Escherichia coli is the main bowel flora. The risk of stool colonization with these species is increased with over three days of antibiotic use and with length of hospital stay, $60 \%$ being colonized by day 15 and over $90 \%$ by day 30 . It was found that colonized babies are the commonest reported source of infection with gut colonization as the major reservoir. ${ }^{28}$

An approach to eradicate potentially diseaseproducing micro-organisms from the oropharynx and gastrointestinal tract of intensive care unit (ICU) patients is selective decontamination of the digestive tract, therefore decreasing the incidence of nosocomial sepsis. A lower cost one is to consider that all babies are colonized and that strict attention to hand washing is routinely encouraged. ${ }^{28}$ Gut decontamination strategies for prevention of septicemia may be needed. ${ }^{33}$

The knowledge about specific risks could allow neonatologist to identify infants at high risk of sepsis and early management. Factors reported to increase the incidence of CRBSI include low birth weight, age below than 7 days, prematurity, number of CVC days, mechanical ventilation and infusion of TPN. ${ }^{14,28,34}$ Previous catheterization was not a significant factor of CRBSI in this study.

In the logistic regression model, birth weight, the duration of catheters in place and those who received TPN remained significant predictors for CRBSI. Mechanical ventilation, age of infection and gestational age were not significant predictors of CRBSI in this model.

Subhani et al. postulated that a higher infection rate in VLBW neonates could reflect functional immaturity of the immune system..$^{35}$ Besides, our results seem to agree with the findings of Gaynes et al., who noted a positive correlation between overall nosocomial infection rates and a measure of device utilization. ${ }^{36}$ This could be explained by the relation between prolonged catheters stay and the higher risk of skin colonization which is associated with an increased risk of CRBSI. ${ }^{37}$ Moreover, TPN need prolongs the duration of CVC stay. ${ }^{13}$ Shouman et al. found that the use of full dose intralipids in TPN decreases the rate of blood stream infection clearance compared to restricted dose of intralipids to $1 \mathrm{~g} / \mathrm{kg} / \mathrm{d}$. Furthermore, TPN adversely affects phagocytosis in neutrophils and increases epithelial permeability. ${ }^{38-40}$

Central venousblood passesthrough reticuloendothelial cells in the lung reaching the peripheral vein which lead to much less concentration of microorganisms in peripheral blood than the catheter blood. This explains exclusion of two cases that do not fulfill this criterion. On the other hand, the barred third case could be explained by the possibility of catheter tip contamination during removal. ${ }^{41}$ 
Limitations to this study are that we did not evaluate several possible confounders such as underlying host factors, antibiotics, crowding, understaffing or severity of illness. Also, surveillance cultures of neonates or the environment were not performed in this study, due to cost and reliability. Finally, we did not categorize the CRBSI rate in our neonatal unit per catheter type.

In summary, this study confirmed that the predictors of CVC infections included low birth weight, prematurity and mechanical ventilation for neonates with HCABSI. This study further elucidated the potential use of TPN, prolonged catheter stay in neonates and low birth weight as independent predictors for CRBSI. Preventive strategies as gut decontamination and limiting the use of invasive devices should be encouraged to minimize $\mathrm{BSI}$ in neonates in our NICU.

\section{References}

1. Centers for Disease Control and Prevention National Healthcare Safety Network Manual, Patient Safety Component Protocol. http://www.cdc.gov/nhsn/PDFs/pscManual/4PSC_ CLABScurrent.pdf; Published 2008. Accessed December 9, 2009.

2. Decker MD, Edwards KM. Central venous catheter infections. Pediatr Clin North Am 1988; 35: 579-612.

3. Baltimore RS. Neonatal nosocomial infections. Semin Perinatol 1998; 22: 25-32. http://dx.doi.org/10.1016/S01460005(98)80005-0

4. Gaynes RP, Edwards JR, Jarvis WR, Culver DH, Tolson JS, Martone WJ. Nosocomial infections among neonates in highrisk nurseries in the United States. National Nosocomial Infections Surveillance System. Pediatr 1996; 98: 357-361.

5. Piedra PA, Dryja DM, LaScolea LJ Jr. Incidence of catheterassociated gram-negative bacteremia in children with short bowel syndrome. J Clin Microbiol 1989; 27: 1317-1319.

6. Mermel LA, Allon M, Bouza E, et al. Clinical practice guidelines for the diagnosis and management of intravascular catheter-related infection: 2009 Update by the Infectious Diseases Society of America. Clin Infect Dis 2009; 49: 1-45. http://dx.doi.org/10.1086/599376

7. Flynn PM. Diagnosis and Management of Central Venous Catheter-Related Bloodstream Infections in Pediatric Patients. Pediatr Infect Dis / 2009; 28: 1016-1017. http://dx.doi. org/10.1097/INF.0b013e3181bf7bfc

8. Ringer SA, Gray JE. Common neonatal procedures. In: Cloherty JP, Eichenwald EC, Stark AR, eds. Manual of Neonatal Care. 6th ed, Philadelphia, Lippincott Williams \& Wilkins, 2008: 660-663.

9. National Committee for Clinical Laboratory Standards Performance Standards for Antimicrobial Disk Susceptibility Tests - Approved Standard. http://openlibrary.org/works/ OL5059073W/Performance_Standards_for_Antimicrobial_ Disk_Susceptibility_Tests-Approved_Standard_(Doc_No._ M2-A5). Published 1993. Accessed October 15, 2012.

10. Sambrook J, Frisch E and Maniatis J. Plasmid vectors. In: Nolan C, Ferguson M, eds. Molecular cloning. A laboratory manual, USA, Cold speing Harbour; 1989: 3-110.
11. Christopher D, Newman PA-C. Catheter-Related Bloodstream Infections in the Pediatric Intensive Care Unit. Semin Pediatr Infect Dis 2006; 17: 20-24. http://dx.doi.org/10.1053/j. spid.2005.11.006

12. O'Grady NP, Alexander M, Dellinger EP, et al. Centers for Disease Control and Prevention. Guidelines for the prevention of intravascular catheter-related infections. MMWR Recomm Resp 2002; 5: 1-29.

13. Tsai M, Hsu J, Lien R, et al. Catheter management in neonates with bloodstream infection and a percutaneously inserted central venous catheter in situ: Removal or not? Am J Inf Cont 2012; 40(1): 59-64. http://dx.doi.org/10.1016/j. ajic.2011.04.051

14. Maas A, Flamentt P, Pardout A, Deplano A, Dramaixg M, Struelens M J. Central venous catheter-related bacteraemia in critically ill neonates: risk factors and impact of a prevention programme. J Hosp Infect 1998; 40: 211-224. http://dx.doi. org/10.1016/S0195-6701(98)90139-6

15. Stover BH, Shulman ST, Bratcher DF, Brady MT, Levine GL, Jarvis WR. Nosocomial infection rates in US children's hospitals' neonatal and pediatric intensive care units. Am J Infect Control 2001; 29: 152-157. http://dx.doi.org/10.1067/ mic.2001.115407

16. Perlman SE, Saiman L, Larson EL. Risk factors for late-onset health care-associated bloodstream infections in patients in neonatal intensive care units. Am / Infect Control 2007; 35: 177-182. http://dx.doi.org/10.1016/j.ajic.2006.01.002

17. Almuneef MA, Memish ZA, Balkhy HH, Hijazi O, Cunningham G, Francis C. Rate, risk factors and outcomes of catheter-related bloodstream infection in a paediatric intensive care unit in Saudi Arabia. J Hosp Infect 2006; 62: 207-213. http://dx.doi.org/10.1016/j.jhin.2005.06.032

18. Edwards JR, Peterson KD, Andrus ML, et al. National Healthcare Safety Network (NHSN) report, data summary for 2006, issued June 2007. American Journal of Infection Control 2007; 35: 290-301. http://dx.doi.org/10.1016/j. ajic.2007.04.001

19. Gupta A, Della-Latta P, Todd B, et al. Outbreak of extendedspectrum beta-lactamase-producing Klebsiella pneumoniae in a neonatal intensive care unit linked to artificial nails. Infect Control Hosp Epidemiol 2004; 25: 210-215. http:// dx.doi.org/10.1086/502380

20. Urrea $M$, Pons $M$, Serra $M$, Latorre $C$, Palomeque $A$. Prospective incidence study of nosocomial infections in a pediatric intensive care unit. Pediatr Infect Dis / 2003; 22: 490493. http://dx.doi.org/10.1097/01.inf.0000069758.00079.d3

21. Richard MJ, Edwards JR, Culver DH, Gaynes RP. Nosocomial infections in pediatric intensive care units in the United States. Pediatrics 1999; 103: e39. http://dx.doi.org/10.1542/ peds.103.4.e39

22. Curry S, Honeycutt M, Goins G, Gilliam C. Catheterassociated blood stream infections in the NICU: Getting to zero. Neonatal network 2009; 28: 151-155. http://dx.doi. org/10.1891/0730-0832.28.3.151

23. Njere I, Islam S, Parish D, Kuna J, Keshtgar AS. Outcome of peripherally inserted central venous catheters in surgical and medical neonates. J Pediatr Surg 2011; 46: 946-950. http:// dx.doi.org/10.1016/j.jpedsurg.2011.02.037

24. O'Grady NP, Alexander M, Dellinger EP, et al. Guidelines for the prevention of intravascular catheter-related infections. Pediatr 2002; 110: e51. http://dx.doi.org/10.1542/ peds.110.5.e51

25. Benjamin Jr DK, Miller W, Garges H, et al. Bacteremia, central catheters, and neonates: when to pull the line. Pediatr 2001; 107: 1272-1276. http://dx.doi.org/10.1542/peds.107.6.1272 
26. Avila-Figueroa C, Goldmann DA, Richardson DK, et al. Intravenous lipid emulsions are the major determinants of coagulase-negative staphylococcal bacteremia in VLBW newborns. Pediatr Infect Dis / 1998; 17: 10-17. http://dx.doi. org/10.1097/00006454-199801000-00004

27. Hsu JF, Tsai M, Huang H, Lien R, Chu S, Huang C. Factors of catheter-related bloodstream infection with percutaneously inserted central venous catheters in VLBW infants: A center's experience in Taiwan. Pediatr Neonatol 2010; 51: 336-342. http://dx.doi.org/10.1016/S1875-9572(10)60065-4

28. KamelYMN, El Nagdy MM, Hawas SA, El Dakar MA, Shouman BO. CD11b, E-Selectin and PCR versus conventional blood culture for diagnosis of neonatal sepsis. EJMM 2012; 21: 3453.

29. Rizk MS, Abo Al-Ela MA, El Dakar MA, Lotfy H. Molecular Typing of Hospital Acquired Klebsiella Isolates from Mansoura University Children Hospital (MUCH). EJMM 2010; 19: 35-43.

30. El-Bayoumi MA, El-Nady GM, Badr R. Clinical and Microbiological study of Nosocomial Infections in the Paediatric Intensive Care Unit (PICU) in Mansoura University Children's Hospital. EJMM 2006; 15: 493-503.

31. Badr RI, Shouman B. Regulated on activation, normal T cell expressed and secreted and tumor necrosis factor-a in septic neonates. J Perinatol 2010; 30: 192-196. http://dx.doi. org/10.1038/jp.2009.167

32. ElMorsy I, Abouelela MA, Hawas SA, Khashaba M. A dramatic change in neonatal Nosocomial infections in NICU, Mansoura University Hospital after strict application of infection control programme. Benha Medical Journal 2010; 27: 661-678.

33. Donnell SC, Taylor N, van Saene HKF, Magnall VL, Pierro A, Lloyd DA. Infection rates in surgical neonates and infants receiving parenteral nutrition: a five year prospective study. J Hosp Infect 2002; 52: 273-280. http://dx.doi.org/10.1053/ jhin.2002.1318
34. Perlman SE, Saiman L, Larson EL. Risk factors for late-onset health care-associated bloodstream infections in patients in neonatal intensive care units. Am I Infect Contro 2007; 35: 177-182. http://dx.doi.org/10.1016/j.ajic.2006.01.002

35. Subhani MT, Dalal J, LaGamma EF. Peripherally inserted central venous catheter (PICC)-related infections correlate with the degree of immaturity rather than operator defined risk factors. Pediatr Res 1998; 43: 197. http://dx.doi. org/10.1203/00006450-199804001-01168

36. Gaynes RP, Martone WJ, Culver DH, et al. Comparison of rates of nosocomial infections in neonatal intensive care units in the United States. Am J Med 1991; 91: 192S-196S. http:// dx.doi.org/10.1016/0002-9343(91)90368-8

37. Hoang V, Sills J, Chandler M, Busalani E, Clifton-Koeppel R, Modanlou HD. Percutaneously inserted central catheter for total parenteral nutrition in neonates: complications rates related to upper versus lower extremity insertion. Pediatr 2008; 121: 1152-1159. http://dx.doi.org/10.1542/peds.20071962

38. Shouman B, Abdelhady H, Badr R, Hammad E, Salama MF Dose of intravenous lipids and rate of bacterial clearance in preterm infants with blood stream infections. Eur / Paediatr 2012; 171: 811-816. http://dx.doi.org/10.1007/s00431-011$1619-y$

39. Donowitz LG, Haley CE, Gregory WW, Wenzel RP. Neonatal intensive care unit bacteremia: emergence of gram-positive bacteria as major pathogens. Am / Infect Contro. 1987; 15: 141-147. http://dx.doi.org/10.1016/0196-6553(87)90137-4

40. Okada Y, Klein NJ, van Saene HK, Webb G, Holzel H, Pierro A. Bactericidal activity against coagulase-negative staphylococci is impaired in infants receiving long-term parenteral nutrition. Ann Surg 2000; 231: 276-281. http:// dx.doi.org/10.1097/00000658-200002000-00018

41. Yagupsky A, Frederick S. Quantitative Aspects of Septicemia. Clin microbiol rev 1990; 3: 269-279. 